\title{
Valor pronóstico del índice de Onodera en la supervivencia del cáncer colorrectal
}

\author{
Prognostic value of Onodera's index in colorectal cancer survival
}

\author{
F. Borda ${ }^{1}$, A. Borda ${ }^{1}$, J.M. Zozaya ${ }^{1}, J$ Urman ${ }^{1}$, J. Jiménez ${ }^{1}$, B. Ibáñez ${ }^{2}$
}

\section{RESUMEN}

Fundamento. El índice de Onodera (IO) combina los valores de los linfocitos circulantes y de la albúmina sérica y se ha utilizado como marcador del estado nutricional, postulándose recientemente en Japón y China su posible valor pronóstico en el cáncer colorrectal. Todavía no disponemos de datos sobre el valor predictivo del IO en una población occidental.

Pacientes y métodos. Se revisaron 207 cánceres colorrectales resecados programada y consecutivamente, con intención curativa. Se calculó el IO pre-tratamiento mediante la fórmula: [ $10 \mathrm{x}$ albúmina sérica $(\mathrm{g} / \mathrm{dl})+0,005$ $\mathrm{x}$ cifra de linfocitos circulantes $/ \mathrm{mm}^{2}$ ]. Consideramos bajos los valores de IO menores de 40 . Se efectuó un análisis univariable y multivariable de las curvas de supervivencia entre los casos con valores de IO menores de 40 (modelo de Cox, stepwise), todo ello en la serie global y en el estadio pTNM II.

Resultados. El seguimiento clínico tuvo una mediana de 81 meses (rango intercuartílico 60-96). Veintiséis pacientes $(12,6 \%)$ presentaron un IO bajo $(<40)$. En el análisis multivariable, los pacientes con IO bajo mostraron unas curvas de supervivencia más desfavorables, tanto en la serie global: $[\mathrm{p}<0,001 ; \mathrm{HR}=3,16$; IC 95\% = 1,67-5,94)] como en los 78 casos en estadio pTNM II: [p $<0,004$; HR $=4,36$; IC $95 \%=1,61-11,76)$ ].

Conclusiones. También en pacientes europeos, un índice de Onodera pre-tratamiento bajo $(<40)$ tiene un valor predictivo independiente y desfavorable sobre la supervivencia en el cáncer colorrectal resecado, tanto en la serie global como en el estadio pTNM II.

Palabras clave. Cáncer colorrectal. Pronóstico pretratamiento. Estadio pTNM II. Indice de Onodera. Índice pronóstico nutricional.

\begin{abstract}
Background. Onodera's prognostic nutritional index (OPNI), which is calculated using total lymphocyte count and serum albumin level, has been used as a marker of nutritional status, with its potential prognostic value in colorectal cancer having recently been postulated in Japan and China. There is still no data on the predictive value of OPNI in a Western population.
\end{abstract}

Patients and methods. A consecutive case series of 207 patients scheduled for colorectal cancer resection with curative intent was reviewed. Pre-treatment OPNI was calculated using the formula: [10 x serum albumin $(\mathrm{g} /$ dl) $+0.005 \times$ lymphocytes $\left./ \mathrm{mm}^{2}\right]$. OPNI values under 40 were considered low. Univariate and multivariate analysis were performed on survival curves, comparing cases with OPNI values less than 40 and the rest of patients (Cox model, stepwise), in the overall series and in pTNM stage II.

Results. The median for clinical follow-up was 81 months (interquartile range 60-96). Twenty-six patients (12.6\%) had a low OPNI $(<40)$. In the multivariate analysis, patients with low OPNI showed less favourable survival curves, both in the overall series: $[p<0.001$; HR $=3.16$; $95 \% \mathrm{CI}=1.67-5.94]$ and in the 78 cases in pTNM stage II: $[p<0.004 ; \mathrm{HR}=4.36 ; 95 \% \mathrm{CI}=1.61-11.76]$.

Conclusions. A low pre-treatment OPNI $(<40)$ has an independent, unfavourable predictive value on survival in European patients with resected colorectal cancer, both in the overall series and in pTNM stage II.

Keywords. Colorectal cancer. Pre-treatment prognosis pTNM stage II. Onodera prognostic nutritional index.
1. Servicio de Digestivo. Complejo Hospitalario de Navarra.

2. NavarraBiomed, Fundación Miguel Servet - Red de Investigación en Servicios Sanitarios en Enfermedades Crónicas (REDISSEC). Pamplona.

Recepción: 5 de febrero de 2014

Aceptación provisional: 23 de abril de 2014

Aceptación definitiva: 10 de junio de 2014

\author{
Correspondencia: \\ Fernando Borda \\ Servicio de Digestivo \\ Complejo Hospitalario de Navarra \\ Irunlarrea, 3 \\ 31008 Pamplona \\ E-mail: bordafernando@yahoo.es
}




\section{INTRODUCCIÓN}

Hoy en día y sumando ambos sexos, el cáncer colorrectal es el tumor maligno que presenta una mayor incidencia en el mundo occidental ${ }^{1}$ y también en nuestra comunidad $^{2}$. A pesar de los continuos progresos efectuados en los tratamientos quirúrgicos y oncológicos, el carcinoma colorrectal sigue presentando una elevada tasa de mortali$\mathrm{dad}^{3,4}$, ocupando el segundo lugar en cuanto a número de fallecimientos por cáncer en España ${ }^{1}$. A diferencia de otras neoplasias digestivas, como el cáncer de páncreas, el carcinoma colorrectal muestra una notable variabilidad en cuanto a su evolución ${ }^{3}$. Por ello, se han estudiado un amplio número de parámetros en relación tanto con las características del paciente como de la neoplasia y de la respuesta inflamatoria sistémica frente al tumor, que pudieran mostrar valor predictivo sobre el pronóstico individual del enfermo, una vez establecido el diagnóstico de la neoplasia ${ }^{5-10}$. En la actualidad se sigue considerando a la estadificación tumoral pTNM $^{11}$ como el "patrón oro" para orientar el pronóstico del cáncer colorrectal, siendo esta clasificación la base para indicar la conveniencia de los tratamientos oncológicos adyuvantes. No obstante, la estadificación pTNM presenta algún inconveniente. En primer lugar, no puede efectuarse en el momento del diagnóstico, requiriendo la resección de la neoplasia y su posterior estudio anatomopatológico. En segundo término, se han registrado diferencias en cuanto a la mortalidad dentro de un mismo estadio tumoral, siendo especialmente destacables en el estadio I $^{11,12}$. Por todo ello, todavía se mantiene la necesidad de disponer de otros parámetros, diferentes al pTNM, para poder predecir la evolución del carcinoma colorrectal y aplicar una terapia más agresiva en los casos con teórico peor pronóstico evolutivo.

Dentro de los numerosos factores que se han estudiado, tanto en forma de parámetros aislados o agrupados en índices pronósticos, se incluye el índice de Onodera (IO), también denominado índice pronóstico nutricional $^{13}$. El IO combina los valores de linfocitos circulantes y albúmina sérica, teniendo por tanto características de marcador nutricional e inmunológico. Los estudios sobre el IO como factor pronóstico en el carcinoma colorrectal son muy escasos ${ }^{13-17}$ y se limitan al área geográfica de Japón y China, no habiendo encontrado en la revisión bibliográfica efectuada ningún trabajo realizado en pacientes occidentales. Dada la influencia de los factores étnicos en el cáncer colorrectal ${ }^{18}$ se ha planteado el presente estudio con el objetivo de analizar en nuestra comunidad el posible valor predictivo del IO, determinado en el momento del diagnóstico del tumor, sobre la supervivencia del carcinoma colorrectal resecado. De modo adicional, se pretende analizar por separado este valor predictivo en el subgrupo de casos en estadio pTNM II, en el cual el pronóstico es más variable y está más discutida la indicación de efectuar un tratamiento adyuvante ${ }^{12}$.

\section{PACIENTES Y MÉTODOS}

Se efectuó un estudio observacional, longitudinal de una serie de 207 carcinomas colorrectales (CCR), registrados de modo consecutivo en un hospital terciario en el periodo junio 2003-junio 2005. Los casos fueron diagnosticados mediante colonoscopia y biopsias endoscópicas. Tras su valoración por el Comité de Oncología Digestiva y siguiendo los protocolos establecidos en nuestro centro, se indicó un tratamiento individualizado, que incluyó la resección quirúrgica con intención curativa, que fue realizada por un mismo equipo, especializado en cirugía colorrectal.

El análisis se centró únicamente en los pacientes intervenidos de forma programada, descartándose los casos operados de urgencia por complicaciones agudas del tumor como obstrucción, hemorragia, abcesificación o perforación. Igualmente se excluyó a un enfermo que falleció dentro de los 30 días siguientes a la operación por considerar el "exitus" debido a una complicación directa de la cirugía. Se efectuó un seguimiento clínico tras el tratamiento, registrándose los casos de fallecimiento debidos al tumor. Para la revisión de las historias clínicas informatizadas de los pacientes, se obtuvo previamente la 
autorización del Comité Ético de nuestro hospital.

El momento de entrada en el estudio, para todos los pacientes, fue el de la confirmación histológica del carcinoma en las biopsias obtenidas endoscópicamente. Las determinaciones analíticas con las que se elaboró el IO fueron las realizadas en la fecha más próxima al inicio del tratamiento: neo-adyuvante o directamente quirúrgico, según la indicación del Cómite de Oncología. En todos los casos la determinación del IO se realizó dentro de un plazo siempre inferior a las dos semanas al comienzo del tratamiento.

Siguiendo las indicaciones de su autor, el índice de Onodera se calculó mediante la fórmula: [10 $\mathrm{x}$ albúmina sérica $(\mathrm{g} / \mathrm{dl})+$ $0,005 \times$ cifra de linfocitos circulantes $\left./ \mathrm{mm}^{2}\right]$ y se consideraron bajos o patológicos los valores del IO menor de $40^{13}$.

Como variables posiblemente relacionadas con el pronóstico del tumor, se consideraron las siguientes: sexo del paciente, edad mayor de 65 años, presencia de cáncer colorrectal sincrónico, grado de diferenciación tumoral: bien diferenciado, medianamente diferenciado e indiferenciado, estadio tumoral pTNM de la American Joint Committe on Cancer $^{11}$, valor del antígeno carcinoembrionario mayor de $6 \mathrm{mg} / \mathrm{l}$ y finalmente índice de Onodera o menor de 40. Efectuamos un análisis univariable de sus curvas de supervivencia mediante los tests de Kaplan Meier y log rank. Las variables que mostraron valor significativo $(\mathrm{p}<0,05)$ fueron incluidas en un análisis multivariable [modelo de Cox, stepwise, calculando el hazard ratio (HR) con un intervalo de confianza del 95\% (IC 95\%)]. Finalmente se determinó la probabilidad de supervivencia a los 3 y 5 años. Estos cálculos se efectuaron tanto para el global de nuestra serie como para los tumores en estadio II.

Los estudios estadísticos se realizaron con los programas R 2.9.2 y SPSS 17 .

\section{RESULTADOS}

Tras la exclusión de un paciente que falleció en los días siguientes a la operación, debido a dehiscencia de sutura, sepsis y fallo multiorgánico, analizamos 207 CCR resecados. De ellos registramos 26 casos $(12,6 \%)$ con un IO bajo $(<40)$. Las características de nuestra serie y su comparación entre los pacientes con IO normal y patológico se recogen en la tabla 1 , no registrándose diferencias significativas entre ambos grupos.

Tabla 1. Características de la serie y diferencias según que el Indice de Onodera sea normal $(\geq 40)$ o bajo $(<40)$

\begin{tabular}{|c|c|c|c|c|}
\hline Variable & $\begin{array}{c}\text { Global } \\
\text { n: } 207 \text { (\%) }\end{array}$ & $\begin{array}{l}\text { I. Onodera } \geq 40 \\
\text { n: } 181(87,4 \%)\end{array}$ & $\begin{array}{c}\text { I. Onodera < } 40 \\
\text { n: } 26(12,6 \%)\end{array}$ & $\mathbf{p}$ \\
\hline Sexo masculino & $121 \quad(58,5)$ & $107(59,1)$ & $14 \quad(53,8)$ & 0,61 \\
\hline Edad $<65$ años & $55(26,6)$ & $51 \quad(28,2)$ & $4 \quad(15,4)$ & 0,17 \\
\hline Cáncer sincrónico & $24(11,6)$ & $19(10,5)$ & $5 \quad(19,6)$ & 0,20 \\
\hline $\begin{array}{l}\text { Grado de diferenciación: } \\
\text { - Bien diferenciado } \\
\text { - Moderadamente diferenciado } \\
\text { - Indiferenciado }\end{array}$ & $\begin{aligned} 40 & (19,3) \\
133 & (64,3) \\
34 & (16,4)\end{aligned}$ & $\begin{aligned} & 34(18,8) \\
& 117(64,6) \\
& 30(16,6) \\
&\end{aligned}$ & $\begin{array}{rr}6 & (23,1) \\
16 & (61,5) \\
4 & (15,4) \\
\end{array}$ & 0,87 \\
\hline $\begin{array}{l}\text { Estadio tumoral pTNM: } \\
\text { - I } \\
\text { - II } \\
\text { - III } \\
\text { - IV }\end{array}$ & $\begin{array}{rr}58 & (28,0) \\
78 & (37,7) \\
59 & (28,5) \\
12 & (5,8) \\
\end{array}$ & $\begin{array}{rr}54 & (29,8) \\
65 & (35,9) \\
53 & (29,3) \\
9 & (5,0)\end{array}$ & $\begin{array}{rr}4 & (15,4) \\
13 & (50,0) \\
6 & (23,1) \\
3 & (11,5) \\
\end{array}$ & 0,18 \\
\hline $\mathrm{CEA}>6 \mathrm{mg} / \mathrm{L}$ & $59 \quad(28,5)$ & $50(27,6)$ & $(34,6)$ & 0,32 \\
\hline
\end{tabular}

CEA: antígeno carcinoembrionario 
El periodo de control clínico tuvo una mediana de 81 meses (rango intercuartílico =60-96). En la tabla 2 se detalla la probabi- lidad de supervivencia de nuestros casos a los 3 y 5 años, tanto en la serie global como en el estadio pTNM II.

Tabla 2. Probabilidad de supervivencia a 3 y 5 años en la serie global y en el subgrupo en estadio pTNM II.

\begin{tabular}{ccc}
\hline & Probabilidad de supervivencia (error estándar) \\
\hline Muestra global & a años 5 años \\
\hline Total & \\
IO $\geq 40$ & $89,8 \%(2,1 \%)$ & $81,4 \%(2,7 \%)$ \\
IO $<40$ & $91,7 \%(2,1 \%)$ & $84,9 \%(2,7 \%)$ \\
\hline Estadio pTNM II & $76,9 \%(8,3 \%)$ & $57,4 \%(9,7 \%)$ \\
\hline Total & & \\
IO $\geq 40$ & $81,5 \%(4,5 \%)$ & $77,0 \%(5,4 \%)$ \\
IO $<40$ & $95,4 \%(2,6 \%)$ & $87,0 \%(4,0 \%)$ \\
\hline
\end{tabular}

$\mathrm{IO}=$ Índice de Onodera.

En la tabla 3 figuran los resultados, correspondientes tanto al global de la serie como al estadio pTNM II, del análisis univariable de la supervivencia para las diversas variables estudiadas, así como las de aquellas que mantienen un valor significativo al efectuar el análisis multivariable.

Tabla 3. Análisis estadístico univariable y multivariable de las curvas de supervivencia en el global de la serie y en el estadio pTNM II

Estadio pTNM II $(\mathbf{n}=\mathbf{7 8})$

\begin{tabular}{|c|c|c|c|c|c|c|}
\hline \multirow{2}{*}{ Variable } & \multicolumn{3}{|c|}{ Análisis univariable } & \multicolumn{3}{|c|}{ Análisis multivariable } \\
\hline & HR & IC (95\%) & $\mathbf{p}$ & HR & IC (95\%) & $\mathbf{p}$ \\
\hline Sexo masculino & 0,90 & $(0,34-2,39)$ & 0,83 & & & \\
\hline Edad $\geq 65$ años & 0,90 & $(0,25-3,15)$ & 0,87 & & & \\
\hline Cáncer sincrónico & 0,67 & $(0,09-5,08)$ & 0,70 & & & \\
\hline Grado de diferenciación & 1,08 & $(0,40-2,89)$ & 0,91 & & & \\
\hline $\mathrm{CEA}>6 \mathrm{mg} / \mathrm{L}$ & 3,26 & $(1,21-8,83)$ & 0,014 & 3,06 & $(1,12-8,37)$ & 0,03 \\
\hline Índice de Onodera < 40 & 4,76 & $(1,77-12,82)$ & 0,001 & 4,36 & $(1,61-11,76)$ & 0,004 \\
\hline
\end{tabular}


Serie global $(\mathbf{n}=\mathbf{2 0 7})$

\begin{tabular}{lcccccc}
\hline \multirow{2}{*}{ Variable } & \multicolumn{3}{c}{ Análisis univariable } & & Análisis multivariable \\
& HR & IC (95\%) & p & HR & IC (95\%) & p \\
\hline Sexo masculino & 1,11 & $(0,62-1,97)$ & 0,730 & & & \\
\hline Edad $\geq 65$ años & 2,86 & $(1,22-6,72)$ & 0,012 & 3,22 & $(1,34-7,77)$ & 0,016 \\
\hline Cáncer sincrónico & 2,81 & $(1,44-5,51)$ & 0,002 & & & \\
\hline Grado de diferenciación & 1,21 & $(0,75-1,95)$ & 0,640 & & & \\
\hline Estadio pTNM: & & & & & & \\
$\quad$ - I & Referencia & & & Referencia & & \\
$\quad$ - II & 3,27 & $(1,09-9,79)$ & & 2,50 & $(0,83-7,53)$ & \\
$\quad$ III & 5,51 & $(1,87-16,22)$ & $<0,001$ & 5,86 & $(1,98-17,32)$ & $<0,001$ \\
- IV & 21,83 & $(6,82-69,88)$ & & 15,24 & $(4,53-51,24)$ & \\
\hline CEA > 6 mg/L & 2,37 & $(1,35-4,17)$ & 0,002 & & & \\
\hline Índice de Onodera $<40$ & 3,46 & $(1,86-6,44)$ & $<0,001$ & 3,16 & $(1,67-5,94)$ & $<0,001$ \\
\hline
\end{tabular}

HR: hazard ratio; IC (95\%): intervalo de confianza al 95\%; CEA: antígeno carcinoembrionario.

La figura 1 representa gráficamente las curvas de supervivencia de los grupos con IO normal y patológico en el global de nuestra casuística. Como puede apreciarse, los pacientes con IO bajo mostraron una evolución significativamente peor que el resto: $\mathrm{p}<0,001 ; \mathrm{HR}=3,16 ;$ IC $95 \%=(1,67-5,94)$.

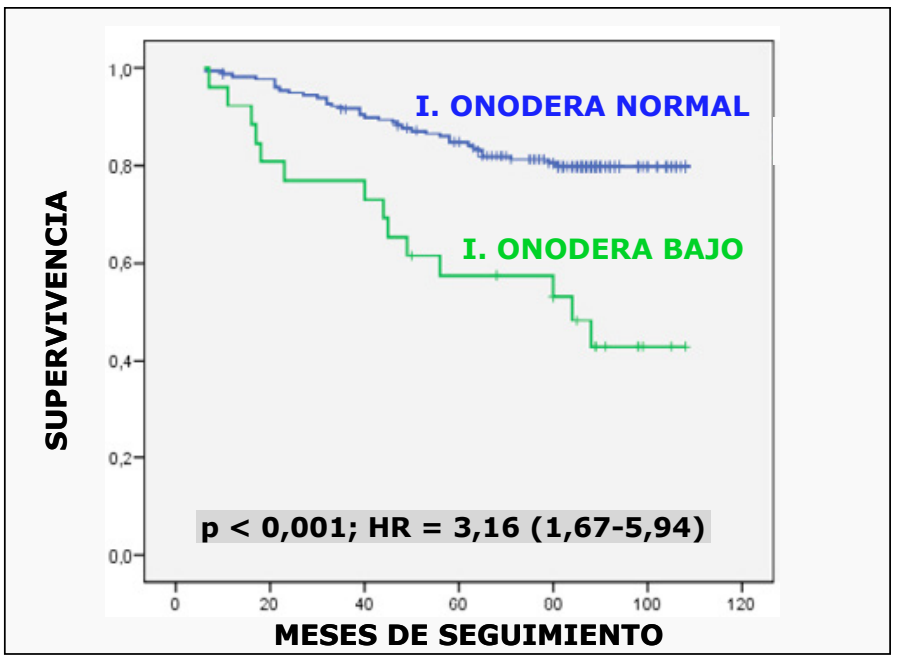

Figura 1. Curvas de supervivencia de la serie global, según su índice de Onodera. 
En nuestra serie, 78 CCR fueron diagnosticados en estadio pTNM II y 13 de ellos $(16,7 \%)$ mostraron un IO < 40. En el análisis univariable el grupo con IO bajo presentó una peor curva de supervivencia $(\mathrm{p}<0,001)$ (Tabla 3), que se confirmó en el estudio multivariable $[\mathrm{p}=0,004 ; \mathrm{HR}=4,36$; IC 95\% = 1,61-11,76)]. (Fig. 2).
Figura 2. Curvas de supervivencia de de los cánceres en estadio pTNM II, según su índice de Onodera.

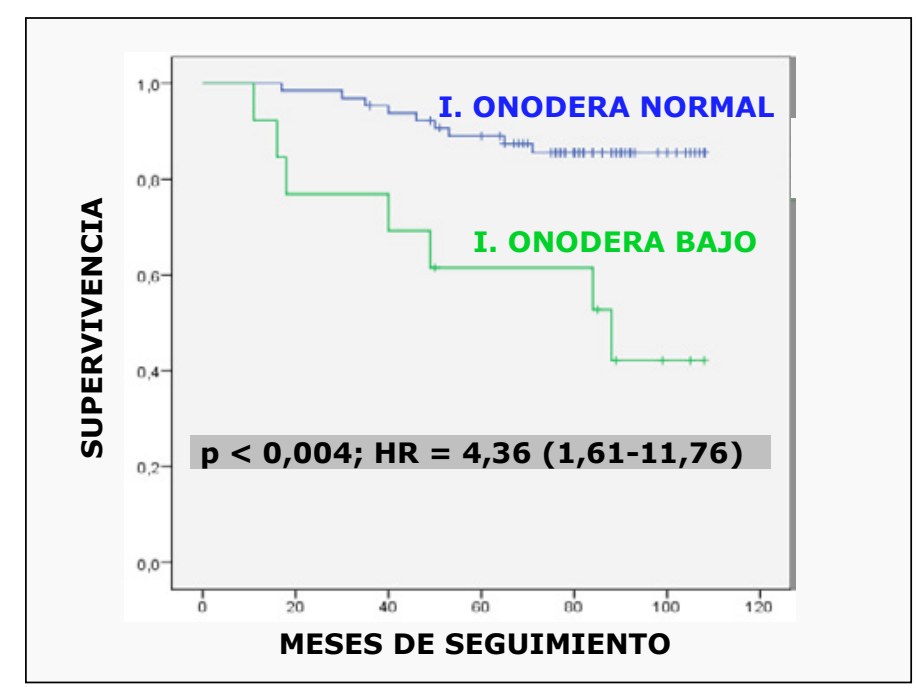

\section{DISCUSIÓN}

El índice de Onodera fue establecido por este autor japonés para valorar el riesgo de presentación de complicaciones en los dos meses siguientes a la resección de cánceres gastrointestinales en pacientes desnutridos ${ }^{13}$, observando peores resultados en los pacientes con un IO menor de 40. Este pronóstico más desfavorable fue confirmado por otros análisis posteriores $^{19-21}$. Además de la mayor morbilidad, se ha postulado que un valor patológico del IO conlleva una mortalidad más alta en diversas neoplasias digestivas como en el cáncer de esófago ${ }^{22}$, estómago ${ }^{23}$, páncreas ${ }^{24}$ e hígado ${ }^{25}$. Aunque algún estudio establece un valor límite para el IO menor de $45^{17,25,26}$ o incluso menor de $47,8^{16}$, nosotros hemos mantenido el punto de corte originalmente propuesto por Onodera: menor de $40^{13}$ y aceptado por la mayoría de autores ${ }^{14,15}$, 19, 24. El valor del IO viene dado por las cifras de albúmina sérica y de linfocitos circulantes. Inicialmente, su poder predictivo se atribuyó a su relación con el estado de nutrición del paciente, por lo que también se le denominó "índice pronóstico nutricional" ${ }^{13}$. En la actualidad, además de su componente de valoración del estado nutritivo, el IO se considera uno de los marcadores de la respuesta inflamatoria sistémica del paciente frente al tumor, ya que en dicha respuesta intervienen tanto la albúmina ${ }^{9}$ como los linfocitos ${ }^{10}$. Por otra parte, también se ha descrito una correlación entre estado nutricional y respuesta inflamatoria en el carcinoma de colon ${ }^{27}$.

Tanto la albuminemia como los linfocitos circulantes han sido analizados en trabajos previos como factores pronósticos de la evolución del cáncer colorrectal, bien de forma aislada ${ }^{10,28} \mathrm{o}$ en combinación con otras variables como el antígeno carcino-embrionario ${ }^{8}$ y la proteína $C$ reactiva (Indice de Glasgow) 5 . Son muy escasas las publicaciones que estudian el posible valor predictivo del IO sobre la supervivencia del carcinoma de colon y recto. Estos 
trabajos son de muy reciente aparición y únicamente se refieren a pacientes de Japón ${ }^{14-17}$ y China $^{26}$. Dos de los estudios se limitan a tumores muy avanzados o recidivados y en ellos un IO bajo presenta un valor pronóstico desfavorable independiente sobre la supervivencia ${ }^{14,16}$, llegando a desaconsejarse la indicación de una cirugía paliativa en los cánceres en estadio IV con IO patológico ${ }^{14}$. Las otras tres publicaciones engloban tumores operados en todos los estadios pTNM y registran una significativa mayor supervivencia para los pacientes con IO pre-quirúrgico normal, frente a los casos con IO patológico ${ }^{15,17,26}$.

Como otros autores, no hemos incluido los cánceres colorrectales que se operaron de modo urgente o con intención puramente paliativa, ya que estos casos presentan de entrada un peor pronóstico y podrían constituir un factor de sesgo a la hora de valorar los resultados ${ }^{29}$. Igualmente se excluyó al paciente fallecido dentro de los primeros 30 días tras la resección, ya que su muerte fue debida a una complicación directa de la cirugía. En nuestra serie, la frecuencia de casos con IO menor de 40 ha sido muy similar a la publicada ${ }^{15}$. Los resultados que hemos obtenido en una muestra representativa de nuestros cánceres colorrectales (formada por casos diagnosticados de modo consecutivo y tratados con iguales criterios), confirman a la estadificación tumoral cómo el "patrón oro" para orientar el pronóstico en esta neoplasia, pero y por primera vez en pacientes no asiáticos, muestran como el registro de un IO patológico en el momento del diagnóstico del carcinoma se sigue de una supervivencia significativamente inferior. En nuestra serie, este valor predictivo es independiente del estadio pTNM, pudiendo orientar el pronóstico antes de tomar la decisión terapéutica y por tanto, sin tener que esperar al estudio de la pieza quirúrgica. Como ventaja adicional, destacaremos que la elaboración del IO se basa en tan solo dos parámetros analíticos séricos, que se efectúan de modo rutinario dentro del estudio de los cánceres de colon y que cumplen los criterios de sencillez, accesibilidad, reproductibilidad y bajo coste.
Ya hemos comentado cómo, en el cáncer colorrectal, se registran diferencias en la supervivencia entre pacientes con un mismo estadio pTNM, siendo dichas diferencias especialmente destacables en estadio pTNM II donde los índices de supervivencia a los 5 años llegan a oscilar en un $30 \%{ }^{11}$. La diversa evolución tras la resección de la neoplasia hace aconsejable buscar otros parámetros predictivos, distintos del estadio tumoral, para poder determinar que subgrupo de pacientes en estadio II presenta un mayor riesgo de progresión o recurrencia tumoral y podría beneficiarse con los tratamientos adyuvantes ${ }^{12}$. Aunque no hay consenso entre las diversas guías clínicas, los criterios más aceptados para este subgrupo serían los anatomopatológicos como crecimiento T4, grado histológico indiferenciado, perforación intestinal y número de adenopatías revisadas menor de $12^{12}$. Junto a estos parámetros "clásicos", se está analizando el posible valor predictivo de diversos biomarcadores: inestabilidad de microsatélites, p53, KRAS, BRAF, interleukina 6 , polimorfismo del gen timidilato-sintasa (TS) etc., sin alcanzarse hasta el momento resultados concluyentes ${ }^{12,30}$.

Por todo ello, hemos querido estudiar de modo selectivo el valor predictivo de la determinación del IO pre-tratamiento en los tumores en estadio pTNM II, dato hasta ahora no referido en la bibliografía. También en este subgrupo de pacientes observamos cómo los casos con IO bajo presentan una supervivencia significativamente inferior al resto, que se mantiene en el análisis estadístico multivariable, por lo que alcanza valor predictivo independiente. Este hallazgo sugiere que los cánceres colorrectales en estadio II y con IO patológico pudieran considerarse como un grupo de mayor riesgo a la hora de valorar la indicación de tratamientos más agresivos.

Como el resto de los trabajos consultados sobre el valor pronóstico a largo plazo del IO en el cáncer colorrectal, nuestro análisis presenta la limitación de ser retrospectivo, lo que disminuye su validez. Igualmente debemos señalar que el valor predictivo analizado se refiere al cáncer re- 
secado, si bien es en este tipo de pacientes donde resulta más útil disponer de variables pronósticas, puesto que en los tumores inoperables o irresecables ya se parte de un pronóstico mucho más desfavorable. Así mismo y al ser un estudio unicéntrico, tanto el número total de tumores como los casos en estadio pTNM II no son muy amplios, lo que puede reducir el poder estadístico de los resultados. El que la serie estudiada provenga de un solo hospital, aunque disminuye la casuística, tiene la ventaja de permitir la homogeneidad del tratamiento quirúrgico, al ser realizado por un único equipo, altamente especializado, lo que evita posibles sesgos dependientes de los cirujanos. Por todo ello y dada la falta de precedentes bibliográficos en nuestra área geográfica, consideramos necesario realizar nuevos estudios, de tipo prospectivo y preferentemente multicéntricos para asegurar la validez externa de nuestros resultados. En caso de confirmarse, el IO constituiría un parámetro sencillo e independiente del estadio tumoral, que facilitaría el pronóstico del cáncer colorrectal resecable, desde el momento del diagnóstico y podría tener utilidad para sentar la indicación de tratamientos oncológicos más agresivos.

\section{BIBLIOGRAFÍA}

1. Morillas JD, Castells A, Oriol I, Pastor A, PérezSegura P, Echevarría JM et al. Alianza para la prevención del cáncer de colon en España: un compromiso cívico con la sociedad. Gastroenterol Hepatol 2012; 35: 109-128.

2. Etxeberria J, Ugarte MD, Barricarte A, Goicoa T, Moreno Iribas C, Azagra MJ et al. Tendencias en las tasas de incidencia de cáncer colorrectal en Navarra en el periodo 1990-2005. An Sist Sanit Navar 2012; 35: 29-39.

3. De Angelis R, Sant M, Coleman MP, Francisci S, Baili P, Pierannunzio D. Cancer survival in Europe $1999-2007$ by country and age: results of EUROCARE-5 a population-based study. The Lancet Oncol 2014; 15: 23-34.

4. Ardanaz E, Moreno-Iribas C, Pérez de Rada ME, EzPonda C, Floristán Y, NavAridas $\mathrm{N}$ et al. Incidencia y mortalidad por cáncer en Navarra. Evolución en los últimos 30 años. An Sist Sanit Navar 2007; 30: 245-270.
5. Roxburg CSD, Crozier JEM, Maxwell F, Foulis AK, Brown J, McKeE RF et al. Comparison of tumour-based (Petersen Index) and inflammation-based (Glascow Prognostic Score) scoring systems in patients undergoing curative resection for colon cancer. $\mathrm{Br} \mathrm{J}$ Cancer 2009; 100: 701-706.

6. Roxburgh CS, McMillan DC. Role of systemic inflammatory response in predicting survival in patients with primary operable cancer. Future Oncol 2010; 6: 149-163.

7. IshizuKa M, Nagata $H$, TAgaKi K, Horie T, Kubota $\mathrm{K}$. Inflammation-based prognostic score is a novel predictor of postoperative outcome in patients with colorectal cancer. Ann Surg 2007; 246: 1047-1051.

8. Boonpipattanapong T, Chewatanakomkul S. Preoperative carcinoembryogenic antigen and albumin in predicting survival in patients with colon and rectal carcinomas. J Clin Gastroenterol 2006; 40: 592-595.

9. Chandra singhe PC, Ediriweera DS, Kumarage SK, DEEn KI. Pre-operative hypoalbuminemia predicts poor overall survival in rectal cancer: a retrospective cohort analysis. BMC Clin Pathol 2013; 13: 12.

10. Chiang SF, Hung HY, Tang R, Changchien CR, Chen JS, You YT et al. Can neutrophil-to-lymphocyte ratio predict the survival of colorectal cancer patients who have recived curative surgery electively? Int J Colorectal Dis 2012; 27: 1347-1357.

11. Edge A, Byrd SB, Compton DR, Fritz CC, GreeNE AG, TrotTI FL. Eds. AJCC (American Joint Committee on Cancer) Cancer Staging Manual, 7th ed. Springer-Verlag, New York 2010.

12. Akiyoshi T, Kobunai T, Watanabe T. Recent approaches to identifying biomarkers for highrisk stage II colon cancer. Surg Today 2012; 42: 1037-1045.

13. Onodera T, Goseki N, Kosaki G. Prognostic nutritional index in gastrointestinal surgery of malnourished cancer patients. Nihon Geka Gakkai Zasshi. 1984; 85: 1001-1005.

14. Maeda K, Shibutani M, Otani H, Nagahara $H$, SugANo K, IKEYA T et al. Low nutricional prognostic index correlates with poor survival in patients with stage IV colorectal cancer following palliative resection of the primary tumor. Word J Surg 2013 Dec 4. [Epub ahead of print].

15. Nozoe T, Kohno M, Iguchi T, Mori E, Maeda T, Matsukuma A et al. The prognostic nutritional index can be a prognostic indicator in colorectal carcinoma. Surg Today 2012 ; 42 : 532535 . 
16. Okada I, Shirahata A, Soda H, Saitou M, Kigawa G, Nemoto H et al. Significance of Onodera's prognostic nutritional index for treating unresectable or recurrent colorectal cancer with chemotherapy. Gan To Kagaku Ryoho 2012; 39: 231-235.

17. Morí Y, Inoue Y, TanaKa K, Hiro J, Uchida K, KusuNOKI M. Prognostic nutritional index predicts postoperative outcome in colorectal cancer. Word J Surg 2013; 37: 2688-2692.

18. LiN YL, LiANG JT. FOLFOX4 in the adyuvant treatment of colon cancer in Asian patients. Hepato Gastroenterology 2012; 59: 400-404.

19. The significance of Onodera's prognostic nutritional index for the treatment of gastrointestinal cancer. Gan To Kagaku Ryoho 2008; 35: 2253-2255.

20. Wakabayashi H, Otani T, Kondo A, Mori S. Reevaluation of the usefulness of the prognostic nutritional index reported by Onodera, especially for the elderly patients undergoing gastrointestinal surgery for the gastric and colon cancer. Japan J Gastroenterol Surg 2004; 37: 472-478.

21. Nozoe T, Kimura Y, Ishida M, Saeki H, Korenaga D. Sugimachi K. Correlation of pre-operative nutritional condition with post-operative complications in surgical treatment for oesophageal carcinoma. Eur J Surg Oncol 2001; 28: 396-400.

22. Asaka S, Naritaka Y, Sagawa M, Shimakawa T, Isohata N, Murayama M et al. A study of nutrition screening for patients with surgically treated esophageal cancer. Gan To Kagaku Ryoho 2009; 36: 1961-1963.

23. Migita K, Takayama T, Saeki K, Matsumoto S, WaKatsuki K, Enomoto K et al. The prognostic nutritional index predicts long-term outcomes of gastric cancer patients independent of tumor stage. Ann Surg Oncol 2013; 20: 2647-2654.

24. Kanda M, Fuji T, Kodera Y, Nagai S, Takeda S, NAKAO A. Nutricional predictors of postoperative outcome in pancreatic cancer. $\mathrm{Br} \mathrm{J}$ Surg 2011; 98: 268-274.

25. Pinato DJ, North BV. Sharma R. A novel, externally validated inflammation-based prognostic algorithm in hepatocellular carcinoma: the prognostic nutritional index. Br J Cancer 2012; 106: 1439-1445.

26. Chang YZ, Cao J, Tan WM, Luo SM, Zhuang SM. Application of Onodera prognostic nutrition index in prognostic evaluation of elderly patients with colorectal cancer. Zhonghua Wei Chang Wai Ke Za Zhi 2013; 16: 561-564.

27. Mauricio SF, da Silva JB, Bering T, Correia Mi. Relationship between nutritional status and the Glascow Prognostic Score in patients with colorectal cancer. Nutrition 2013; 29: 625-629.

28. Lai CC, You JF, Yeh CY, Chen JS, Tang R, Wang JY. Low preoperative serum albumin in colon cancer: a risk factor of poor outcome. Int $\mathrm{J}$ Colorectal Dis 2011; 26: 473-481.

29. Dixon MR, Haukoos HS, Udani SM, Naghi JJ, ARNELL TD, KUMAR RR Et al. Carcinoembryonic antigen and albumin predict survival in patients with advanced colon and rectal cancer. Arch Surg 2003; 138: 962-926.

30. Nitsche U, Rosenberg R, Balmert A, Schuster T, Slotta-Huspenina J, Herrmann $P$ et al. Integrative marker análisis allows risk assessment for metastasis in stage II colon cancer. Ann Surg 2012; 256: 763-771. 